\section{Initial management of ocular \\ hypertension and primary open-angle glaucoma: an evaluation of the royal college of ophthalmologists' guidelines}

\begin{abstract}
Purpose The purpose of this study was to identify any consensus of opinion among consultant ophthalmologists in Wales with respect to the initial management of glaucoma referrals based on the published guidelines of the Royal College of Ophthalmologists (RCO) and to compare consultant opinion with the practice in a typical hospital.

Method The RCO guidelines document was studied to identify clear statements, which could be adopted as standards for audit purposes. A questionnaire was designed and sent to all consultant ophthalmologists in Wales $(n=37)$ to obtain their opinions. An audit was performed of $\mathbf{1 0 0}$ consecutive patients referred to our unit as glaucoma suspects with regard to initial management. Descriptive statistical analysis was performed. Results A good response rate for a postal questionnaire was obtained $(81 \%)$ with $79.1 \%$ of responders finding the guidelines of at least some help. Levels of agreement with the definitions of ocular hypertension $(\mathrm{OH})$ and primary open-angle glaucoma (POAG) were 76.7 and $86.7 \%$, respectively. There was consensus of consultant opinion regarding many of the elements of the baseline clinical assessment with the significant exceptions of the necessity for dilated fundoscopy, gonioscopy, retinal nerve fibre layer assessment, and drawing of optic discs. The various 'clinical scenarios' for management of the RCO document were not all endorsed. The clinical audit results of the initial management
\end{abstract}

YF Choong, N Devarajan, A Pickering, S Pickering and MW Austin

of glaucoma referrals accurately reflected the consensus of the consultant opinion.

Discussion The RCO guidelines document represents a useful contribution to the management of patients with $\mathrm{OH}$ and POAG. Nevertheless, considerable variation in opinion exists concerning even the most basic areas. With the advent of clinical governance, bridging the gap between the conclusions of College working parties and realities of everyday practice will assume greater importance.

Eye (2003) 17, 685-690. doi:10.1038/

sj.eye. 6700633

Keywords: glaucoma; ocular hypertension; diagnosis; management; guidelines

\section{Introduction}

The management of patients with ocular hypertension $(\mathrm{OH})$ and primary open-angle glaucoma (POAG) accounts for a significant proportion of the workload of most ophthalmologists. ${ }^{1}$ This includes consultants with a special interest in glaucoma and those with other special interests who see patients with glaucoma in general clinics. Although there is a large amount of published data on the diagnosis, pathophysiology, epidemiology, and treatment of $\mathrm{OH}$ and POAG, there exists a wide body of opinion concerning screening, referral, management and best practice. ${ }^{1-13}$ Therefore, the Royal College of Ophthalmologists (RCO)
Department of Ophthalmology Singleton Hospital Sketty

Swansea, UK

Correspondence: MW Austin

Department of Ophthalmology Singleton Hospital, Sketty Swansea SA2 8QA, UK Tel: + 441792285036 Fax: +441792 208647

E-mail: maustin@ globalnet.co.uk

Received: 21 November 2002

Accepted: 24 April 2003

The authors have no proprietary interests or research funding in any products mentioned in this manuscript

This paper was presented in part at the Annual Congress of the Royal College of Ophthalmologists, Birmingham, May 2001 
guidelines ${ }^{14}$ for the management of $\mathrm{OH}$ and POAG should be welcomed. The guidelines' authors, on behalf of the RCO, have clearly stated that their document represents consensus guidelines and is not to be considered a protocol for management. As with many treatment or management guidelines published by various professional bodies, there is little data on the dissemination and acceptance of these guidelines by clinicians in practice. Information on their usefulness, compliance, and the causes of noncompliance is sparse. Although there is evidence for a high rate of conformance with recommended patterns of care for glaucoma patients in an academic, public clinic setting, that is, a specialist clinic setting, ${ }^{4}$ conformance is lower within the private, community-based setting, that is, nonspecialist clinic setting ${ }^{7}$ (in the USA).

The purpose of this study was to survey the consensus of opinion among consultant ophthalmologists in Wales with regard to the published guidelines of the $\mathrm{RCO}^{14}$ and to compare with audited departmental practice with respect to the initial management of glaucoma referrals.

\section{Materials and methods}

\section{Survey of consultant ophthalmologists}

The RCO guidelines document for the management of ocular hypertension $(\mathrm{OH})$ and primary open-angle glaucoma (POAG) was studied to identify what were felt to be clear statements about initial management, which could be adopted as standards for audit purposes. These were (Table 2); unconfirmed or temporary elevations of intraocular pressure (IOP) (isolated finding) should result in discharge; persistently elevated IOP should result in follow-up; IOP $>30 \mathrm{mmHg}$ should be treated as should IOP $>25 \mathrm{mmHg}$ in the presence of asymmetry in optic disc cupping of $>0.2$. A self-administered questionnaire was designed incorporating the standards identified and sent by post to all consultant ophthalmologists in Wales $(n=37)$ to obtain their opinions.

\section{Departmental audit (glaucoma care pathway)}

All patients referred to our unit who require a glaucoma assessment are initially seen by an ophthalmic nurse practitioner (ONP). During this visit, a standardised medical and ophthalmic history is taken, which includes risk factors for glaucoma. IOP is measured using Goldmann applanation tonometry and automated visual fields (Humphrey Visual Field Analyser 24-2 Fastpac) performed. An ophthalmologist will see each patient at the next visit. Working to an agreed protocol, those judged by the ONP to have high risk of glaucoma will have their appointment expedited and are seen within 2 months, either immediately (IOP $\geqslant 30 \mathrm{mmHg}$ or severe field defect) or in the next available clinic. Those with a lower risk receive a follow-up appointment within 3 months. In this way, patients with a high risk of glaucoma will be able to see an ophthalmologist promptly and be commenced on treatment as indicated.

A case-note audit of the initial management of a prospective series of patients referred for glaucoma assessment was performed. Data were collected from 100 consecutive patients using the standards identified in the RCO guidelines as surveyed in the consultants' questionnaire and recorded in Tables 2 and 3. This audit of departmental practice in Swansea concerned both the initial assessment with the ONP and the following visit to an ophthalmologist of middle-grade standing or above in the outpatient clinic. Descriptive analyses were performed on the data collected using MS Excel 2000 (Microsoft Corporation, Seattle, WA, USA).

\section{Results}

\section{Questionnaire survey}

A good response rate for a postal questionnaire was obtained with 30 of $37(81.1 \%)$ consultants returning their questionnaires. Of those responding, 30\% specified a special interest in glaucoma (Table 1). Although most knew about the existence of the guidelines, $14.3 \%$ (four of 28) had not read them. Most respondents found the guidelines of some help but a substantial minority (20.8\%) did not (Table 1).

Agreement with the definitions of $\mathrm{OH}$ and POAG were 76.7 and $86.7 \%$, respectively, with a substantial minority disagreeing (Table 2). Generally, there was a high level of agreement between the elements of baseline assessment recommended in the RCO guidelines and the consultants' opinions. It is noteworthy however that

Table 1 Questionnaire survey of consultant ophthalmologists' opinion regarding the RCO guidelines for the management of glaucoma and $\mathrm{OH}$

\begin{tabular}{|c|c|c|}
\hline & No. & $\%$ \\
\hline $\begin{array}{l}\text { Number of consultant ophthalmologists } \\
\text { in Wales (June 2000) }\end{array}$ & 37 & 100.0 \\
\hline Number of questionnaire respondents & 30 & 81.1 \\
\hline $\begin{array}{l}\text { Number of respondents with a special } \\
\text { interest in glaucoma }\end{array}$ & 9 of 30 & 30.0 \\
\hline Number aware of the college guidelines & 28 of 30 & 93.3 \\
\hline Number who had read the guidelines & 24 of 28 & 85.7 \\
\hline \multicolumn{3}{|l|}{ Number who find the guidelines } \\
\hline (a) Helpful & 5 of 24 & 20.8 \\
\hline (b) Of some help & 14 of 24 & 58.3 \\
\hline (c) Not helpful & 5 of 24 & 20.8 \\
\hline
\end{tabular}


Table 2 Agreement with the RCO guidelines definitions and management of the various clinical scenarios

\begin{tabular}{|c|c|c|c|c|}
\hline & \multicolumn{3}{|c|}{$\begin{array}{l}\text { Questionnaire survey of } \\
\text { consultant ophthalmologists in Wales }(\mathrm{n}=30)\end{array}$} & \multirow{2}{*}{$\begin{array}{l}\text { Audit patients having } \\
\text { each management } \\
\text { intervention }(\mathrm{n}=100)\end{array}$} \\
\hline & Agree & Disagree & Neither & \\
\hline \multicolumn{5}{|l|}{ Definitions } \\
\hline Ocular hypertension $(\mathrm{OH})^{\mathrm{a}}$ & $23(76.7 \%)$ & $7(23.3 \%)$ & - & - \\
\hline Primary open-angle glaucoma $(\mathrm{POAG})^{\mathrm{b}}$ & $26(86.7 \%)$ & $4(13.3 \%)$ & - & - \\
\hline \multicolumn{5}{|l|}{ Guidelines for $\mathrm{OH}$ management } \\
\hline $\begin{array}{l}\text { 'Unconfirmed' elevated IOP should be } \\
\text { discharged }\end{array}$ & $26(86.7 \%)$ & $4(13.3 \%)$ & - & $\begin{array}{c}15(37.5 \%) \\
n=40\end{array}$ \\
\hline 'Temporary' elevated IOP should be discharged & $28(93.3 \%)$ & $2(6.7 \%)$ & - & \\
\hline $\begin{array}{l}\text { 'Persistent' elevated IOP should be followed } \\
\text { up routinely }\end{array}$ & $16(53.3 \%)$ & $14(46.7 \%)$ & - & $11(100 \%)$ \\
\hline Treat IOP $\geqslant 30 \mathrm{mmHg}$ & $28(93.3 \%)$ & $1(3.3 \%)$ & $1(3.3 \%)$ & $\begin{array}{l}2(100 \%) \\
n=2\end{array}$ \\
\hline Treat IOP $>25$ with cup : disc asymmetry $>0.2$ & $8(26.7 \%)$ & $15(50.0 \%)$ & $7(23.3 \%)$ & $0^{c}$ \\
\hline
\end{tabular}

Table 3 Agreement with the suggested elements of a baseline assessment for possible glaucoma and $\mathrm{OH}$

\begin{tabular}{|c|c|c|c|c|}
\hline & \multicolumn{3}{|c|}{ Survey of consultants $(\mathrm{n}=30)$} & \multirow{2}{*}{$\begin{array}{l}\text { Audit patients having } \\
\text { each item }(\mathrm{n}=100)\end{array}$} \\
\hline & Agree & Disagree & Neither & \\
\hline Past ophthalmic history & $28(93.3 \%)$ & $2(6.7 \%)$ & 0 & 100 \\
\hline Presence/absence of family history & $29(96.7 \%)$ & 0 & $1(3.3 \%)$ & 100 \\
\hline Presence/absence of high myopia & $27(90.0 \%)$ & 0 & $3(10.0 \%)$ & 100 \\
\hline Presence/absence of diabetes & $26(86.7 \%)$ & 0 & $4(13.3 \%)$ & 100 \\
\hline IOP measurement by applanation & $100(100.0 \%)$ & 0 & 0 & 100 \\
\hline Documented anterior segment examination & $29(96.7 \%)$ & 0 & $1(3.3 \%)$ & 93 \\
\hline Gonioscopy & $21(70.0 \%)$ & $1(3.3 \%)$ & $8(26.7 \%)$ & 23 \\
\hline Slit-lamp biomicroscopic disc assessment & $27(90.0 \%)$ & $1(3.3 \%)$ & $2(6.7 \%)$ & 100 \\
\hline Cup: disc ratio & $27(90.0 \%)$ & $2(6.7 \%)$ & $1(3.3 \%)$ & 71 \\
\hline Disc drawing & $25(83.3 \%)$ & $1(3.3 \%)$ & $4(13.3 \%)$ & 84 \\
\hline Dilated fundoscopy should be the norm & $19(63.3 \%)$ & $2(6.7 \%)$ & $9(30.0 \%)$ & 63 \\
\hline Visual fields analysis & $28(93.3 \%)$ & $1(3.3 \%)$ & $1(3.3 \%)$ & 100 \\
\hline
\end{tabular}

lower levels of agreement were observed with respect to pupil dilation for stereoscopic optic disc assessment $(63.3 \%)$, gonioscopy $(70 \%)$, and drawing of optic discs $(83.3 \%)$ (Table 3$)$.

With regard to the management of $\mathrm{OH}, 86.7 \%$ agreed that 'unconfirmed' $\mathrm{OH}$ and $93.3 \%$ that 'temporary' $\mathrm{OH}$ should be discharged to the optometrists. Only $53.3 \%$ of respondents agreed that 'persistent' $\mathrm{OH}$ should be routinely followed up in a hospital clinic. Most (93.3\% of the respondents) agreed that the clinical scenario of 'IOP consistently $30 \mathrm{mmHg}$ or above' should be treated. Only $26.7 \%$ agreed that treatment is indicated for a clinical scenario of 'IOP consistently over $25 \mathrm{mmHg}$ in the presence of a cup: disc ratio (CDR) asymmetry of 0.2 or more,' whereas $23.3 \%$ could not bring themselves to agree or disagree with the scenario (Table 2). This indicated a degree of ambivalence towards this guideline which questions its usefulness.

\section{Departmental audit}

A total of 100 consecutive case notes of patients referred for possible glaucoma or $\mathrm{OH}$ were obtained and reviewed by the authors. Considering the combined efforts of the ONP and the ophthalmologists, there was $100 \%$ compliance with the RCO guidelines recommendation for baseline assessment in most categories (see Table 3). Significant exceptions were 
adequate documentation of the anterior segment examination $(93 \%)$, gonioscopy $(23 \%)$, dilated fundoscopy (63\%), CDR (71\%), and disc drawing (84\%), that is, those elements of clinical examination where consensus of consultant opinion was most lacking.

Visual fields were performed in all cases, of which $42 \%$ were equivocal and unhelpful. Causes of equivocal fields were high false-positive errors (12\%), high false-negative errors $(16 \%)$, high fixation losses $(48 \%)$, a pattern of the 'defect' not matching the disc findings (41\%), and artefacts $(60 \%)$, that is, these initial visual fields were unhelpful in generating information useful in the clinical evaluation of any glaucomatous optic neuropathy.

A diagnosis of glaucoma was made in $24 \%$. A total of $40 \%$ were thought to be normal and $33 \%$ were labelled as suspect/OH. With regard to the RCO guidelines for management of $\mathrm{OH}$ that states that 'unconfirmed' $\mathrm{OH}$ and 'temporary' $\mathrm{OH}$ should be discharged to the optometrists, our audit showed that only $38 \%$ of those in this category were discharged in practice. In contrast, 86.7 and $93.3 \%$, respectively, of consultants in the survey agreed that such patients should be discharged. All confirmed $\mathrm{OH}$ were followed up in our clinic, which was in keeping with the RCO guidelines. The RCO guidelines also recommend that the clinical scenario of 'intra-ocular pressure (IOP) consistently $30 \mathrm{mmHg}$ or above' should be treated. There were only two patients in the audit group who fell into this category and both were started on treatment at their first doctor visit. None of the patients in the audit was in the clinical scenario of 'IOP consistently over $25 \mathrm{mmHg}$ and a cup: disc ratio (CDR) asymmetry of 0.2 or more'. A total of $58 \%$ were given follow-up appointments but did not receive any treatment, $27 \%$ were commenced on topical treatment (70\% beta-blocker and $30 \%$ latanoprost), and only $10 \%$ were seen by the ONP for their next visit after seeing the ophthalmologist, which would have been for an essentially 'IOP only' consultation.

\section{Discussion}

Clinical guidelines are produced by professional bodies such as the RCO in order to confirm consensus as to the nature of important diseases, their defining characteristics, and the evidence base for their management. Such guidelines aim to alter standards of care for the better by allowing all clinicians to be aware of what is considered to be the current best practice. As part of RCO guidelines production, the results of expert working parties are circulated in draft form to all consultant ophthalmologists prior to final publication. Such a set of guidelines was produced for $\mathrm{OH}$ and glaucoma in 1997. To our knowledge, these guidelines have not been the subject of any kind of audit as to how they have been received and how they operate in day-today practice. These issues formed the rationale for our study. It was undertaken during a period of review of the RCO guidelines for the management of $\mathrm{OH}$ and POAG and prior to the publication of the $\mathrm{OH}$ treatment study. ${ }^{15}$ Thus, it is possible that our results and conclusions may require consideration in the light of any significant amendments to the guidelines arising as a result of revision and the findings of such recent and ongoing research that will inevitably influence future decisionmaking in $\mathrm{OH}$ and glaucoma.

Most glaucoma care takes place outside of specialist glaucoma clinics. It was for this reason that we chose to study the opinions and practices of consultants in general and 'general' rather than only 'special interest' clinics. We feel that the RCO guidelines represent a useful contribution to the management of patients with $\mathrm{OH}$ and POAG. Nevertheless, considerable variation in opinion exists concerning even the most basic areas. These include the definitions of $\mathrm{OH}$ and POAG. Most respondents to the questionnaire agreed to the suggested management of discharging 'unconfirmed' and 'temporary' $\mathrm{OH}$. This was not mirrored by clinical practice in our department, resulting in a significant number of patients being followed up, probably unnecessarily, possibly because of clinicians' reluctance to make a decision. Given the pressure of numbers of referrals to the service, this reluctance to discharge is of concern. The questionnaire respondents and the clinical practice of our department endorsed the treatment of $\mathrm{OH}$ with IOP of $30 \mathrm{mmHg}$ or more but not the other 'highrisk' $\mathrm{OH}$ scenario (IOP consistently over $25 \mathrm{mmHg}$ and a CDR asymmetry of greater than 0.2 ).

Since glaucoma is a progressive disease, sequential data on IOP, visual fields, and disc appearance are vital in making a diagnosis and monitoring disease progression. ${ }^{16-20}$ Thus a detailed baseline assessment is essential. There was consensus in the consultant survey regarding many of the elements of the baseline assessment as recommended in the guidelines with significant exceptions of stereoscopic disc assessment, gonioscopy, and drawing of optic discs. It is of some concern that consultant ophthalmologists are unable to agree regarding the best methods of assessment required for making a diagnosis of glaucoma, namely evaluation and recording of optic disc appearance and configuration of anterior chamber drainage angle. The importance of a good baseline assessment should be emphasised especially to junior medical staff who should be taught gonioscopy techniques both to recognise the normal and characterise the pathological.

It is well recognised that ophthalmoscopic and biomicroscopic optic disc assessment is largely subjective and is subject to inherently high inter- and intraobserver 
variation..$^{21-24}$ In addition, documentation of disc appearance by nonexpert free-hand drawing is difficult, inaccurate, and often poor. We would advocate that both stereoscopic disc imaging and scanning laser ophthalmoscopy be made more widely available for routine clinical use following their validation in research settings. ${ }^{22-30}$

In order to streamline clinics and improve continuing care, most patients requiring an 'IOP-only check' following commencement of treatment can be seen by a specialist nurse or glaucoma technician at their next visit rather than by the ophthalmologist thus freeing up doctor appointment slots. An important role of such individuals with their proven ability to work to guidelines and protocols may be seen as being 'guardians of the data' - ensuring that IOP measurements, reliable visual field test results, and disc imaging are collated in the patients' records.

In conclusion, the RCO document is a helpful initial step to providing guidelines for the assessment and management of $\mathrm{OH}$ and POAG. Some useful auditable standards identified from the document are presented in this study. However, significant disagreement exists about these standards as seen in our survey and departmental audit. This reflects the complexity and the variation of the disease and its management together with the glaucoma workload being greater than the capacity of specialist glaucoma clinics in most units. Further clarification and revision of the guidelines may be required if they are to be accepted outside the practice of the glaucoma enthusiasts. With the advent of clinical governance, bridging the gap between the conclusions of RCO working parties and realities of everyday practice will assume greater importance.

\section{References}

1 Harrison RJ, Wild JM, Hobley AJ. Referral patterns to an ophthalmic outpatient clinic by general practitioners and ophthalmic opticians and the role of these professionals in screening for ocular disease. BMJ 1988; 297(6657): 1162-1167.

2 Tuck MW, Crick RP. Efficiency of referral for suspected glaucoma. BMJ 1991; 302(6783): 998-1000.

3 Sommer A, Weiner JP, Gamble L. Developing specialty-wide standards of practice: the experience of ophthalmology. QRB Qual Rev Bull 1990; 16(2): 65-70.

4 Albrecht KG, Lee PP. Conformance with preferred practice patterns in caring for patients with glaucoma. Ophthalmology 1994; 101(10): 1668-1671.

5 Cioffi GA. Care guidelines and optic nerve assessment. J Glaucoma 1996; 5(1): A12.

6 Craven ER. Risk management issues in glaucoma: diagnosis and treatment. Surv Ophthalmol 1996; 40(6): 459-462.

7 Hertzog LH, Albrecht KG, LaBree L, Lee PP. Glaucoma care and conformance with preferred practice patterns.
Examination of the private, community-based ophthalmologist. Ophthalmology 1996; 103(7): 1009-1013.

8 Heijl A. A national glaucoma care program. Acta Ophthalmol Scand 1997; 75(3): 295-298.

9 Lee PP. Understanding the new primary open-angle glaucoma preferred practice pattern. Int Ophthalmol Clin 1998; 38(3): 93-99.

10 Stamper RL. Glaucoma screening. J Glaucoma 1998; 7(3): 149-150.

11 Vernon SA, Ghosh G. Do locally agreed guidelines for optometrists concerning the referral of glaucoma suspects influence referral practice? Eye 2001; 15(Part 4): 458-463.

12 Serle J, Cantor L, Gross R, Katz J, Mundorf T, Noecker R et al. Best practice treatment algorithm for primary openangle glaucoma: implications for U.S. ophthalmology practice. Manage Care Interface 2002; 15(7): 37-48.

13 American Academy of Ophthalmology. Primary open angle glaucoma suspect. In: American Academy of Ophthalmology (AAO) National Guidelines. American Academy of Ophthalmology: San Francisco, CA, 2000

14 Vernon S, Hugkulstone C, Jay J, Menage M, Fielder A. Guidelines for the Management of Ocular Hypertension and Primary Open Angle Glaucoma. The Royal College of Ophthalmologists: London, 1997.

15 Kass MA, Heuer DK, Higginbotham EJ, Johnson CA, Keltner JL, Miller JP et al. The ocular hypertension treatment study. Arch Ophthalmol 2002; 120: 701-713.

16 The advanced glaucoma intervention study (AGIS): 7 . The relationship between control of intraocular pressure and visual field deterioration. The AGIS Investigators. Am J Ophthalmol 2000; 130(4): 429-440.

17 Gloster J. Quantitative relationship between cupping of the optic disc and visual field loss in chronic simple glaucoma. Br J Ophthalmol 1978; 62(10): 665-669.

18 Caprioli J, Miller JM, Sears M. Quantitative evaluation of the optic nerve head in patients with unilateral visual field loss from primary open-angle glaucoma. Ophthalmology 1987; 94(11): 1484-1487.

19 Gloster J. Quantitative studies of visual field loss and cupping of the optic disc: their relevance to the management of chronic simple glaucoma. Trans Ophthalmol Soc UK 1979; 99(1): 82-83.

20 Wilson R, Walker AM, Dueker DK, Crick RP. Risk factors for rate of progression of glaucomatous visual field loss: a computer-based analysis. Arch Ophthalmol 1982; 100(5): 737-741.

21 Abrams LS, Scott IU, Spaeth GL, Quigley HA, Varma R. Agreement among optometrists, ophthalmologists, and residents in evaluating the optic disc for glaucoma. Ophthalmology 1994; 101(10): 1662-1667.

22 Hanson S, Krishnan SK, Phillips J. Observer experience and cup : disc ratio assessment. Optometry Vis Sci 2001; 78(10): 701-705.

23 Hatch WV, Trope GE, Buys YM, Macken P, Etchells EE, Flanagan JG. Agreement in assessing glaucomatous discs in a clinical teaching setting with stereoscopic disc photographs, planimetry, and laser scanning tomography. J Glaucoma 1999; 8(2): 99-104.

24 Tielsch JM, Katz J, Quigley HA, Miller NR, Sommer A. Intraobserver and interobserver agreement in measurement of optic disc characteristics. Ophthalmology 1988; 95(3): 350-356.

25 Shuttleworth GN, Khong CH, Diamond JP. A new digital optic disc stereo camera: intraobserver and interobserver 
repeatability of optic disc measurements. Br J Ophthalmol 2000; 84(4): 403-407.

26 Ikram MK, Borger PH, Assink JJ, Jonas JB, Hofman A, de Jong PT. Comparing ophthalmoscopy, slide viewing, and semiautomated systems in optic disc morphometry. Ophthalmology 2002; 109(3): 486-493.

27 Jonas JB, Mardin CY, Grundler AE. Comparison of measurements of neuroretinal rim area between confocal laser scanning tomography and planimetry of photographs. Br J Ophthalmol 1998; 82(4): 362-366.
28 Sung VC, Bhan A, Vernon SA. Agreement in assessing optic discs with a digital stereoscopic optic disc camera (Discam) and Heidelberg retina tomograph. Br J Ophthalmol 2002; 86(2): 196-202.

29 Spencer AF, Sadiq SA, Pawson P, Vernon SA. Vertical optic disk diameter: discrepancy between planimetric and SLO measurements. Invest Ophthalmol Vis Sci 1995; 36(5): 796-803.

30 Greenfield D. Optic nerve and retinal nerve fiber layer analyzers in glaucoma. Curr Opin Ophthalmol 2002; 13: 68-76. 